\title{
COHERENT AND SEQUENTIAL TUNNELING IN HIGH MAGNETIC FIELD
}

\author{
A.E. Belyaev, S.A. Vitusevich \\ Institute of Semiconductor Physics, National Academy of Sciences of Ukraine \\ Prospekt Nauki 45, 252028 Kiev, Ukraine \\ D. Maude and J.C. Portal
}

Service National des Champs Intenses

Centre National de la Recherche Scientifique, 38042 Grenoble, France

In the resonant tunneling diode incorporating a wide one-sided spacer layer, an oscillation picture has been studied in both polarities of the applied voltage in high magnetic field. The results lead to the conclusion that the interference between the electron waves running in the forward direction and the ones reflected at some potential step can occur in both the emitter and collector regions. The characteristic lengths corresponding to the path of the ballistic motion of electrons were estimated. An exchange enhancement of the electronic $g$-factor in two-dimensional systems was observed.

PACS numbers: 73.40.Gk, 85.30.Mn

\section{Introduction}

In our previous papers we have reported the study of the fine periodic structure in the tunnel current flowing through resonant-tunneling devices under the resonance conditions. The effect was satisfactorily described in terms of the model of quantum interference of ballistic electrons [1]. Thus, the fact of observation of the tunnel current oscillations could be used as a probe to distinguish the mechanisms by which the tunneling occurs, i.e., sequential or coherent tunneling. To answer this question we have to know the regions exactly, where the interference between the electron waves running in the forward direction and the ones reflected at some potential step occurs. Magnetotunneling measurements have been widely used to analyse the dimensionality of the emitter electron gas of resonant tunneling diode [2]. Since the dimensionality of the emitter electron gas has a profound effect on the fine periodic structure in the tunnel current, the magnetic-field-induced changes in the current-voltage characteristics (CVC) could help to determine the regions where the interference takes place. The latter statement is valid in the case of high enough magnetic fields providing the magnetic length is smaller or 
comparable with the one of the characteristic kinetic lengths (mean free path, energy relaxation length, etc.). In this work, we have examined the behaviour of the tunnel current oscillations as well as changes of the CVC in high magnetic fields (up to $25 \mathrm{~T}$ ) applied in both parallel and perpendicular directions to the current flow.

\section{Experimental results and discussion}

The magnetotunneling measurements have been carried out on a series of GaAs/AlAs double barrier devices with varying contact and spacer layers. The active part of double-barrier heterostructures (DBRH) consisted of two very thin (2 nm for DBRH-1 and 1.7 for DBRH-2) AlAs barriers separated by a GaAs quantum well ( $4 \mathrm{~nm}$ for DBRH-1 and $5.6 \mathrm{~nm}$ for DBRH-2). A wide spacer layer (undoped GaAs, $100 \mathrm{~nm}$ width) was grown between the barrier and doped contact on the one side of the structure DBRHI-1. In the case of DBRHI-2 the top spacer layer comprised undoped GaAs of $2 \mathrm{~nm}$ width followed by lightly doped $\left(10^{17} \mathrm{~cm}^{-3}\right) \mathrm{GaAs}$ of a $70 \mathrm{~nm}$ width. The bottom spacer layer consisted of undoped $\left(2 \times 10^{16} \mathrm{~cm}^{-3}\right) \mathrm{GaAs}$ of a $1 \mu \mathrm{m}$ width. The results obtained on both structures were very similar. Thus, we will discuss the experimental results obtained on the structure DBRII-1 only. Two quasi-bound states of the well are expected to appear in such structures and, really, two resonant peaks were observed in both polarities of applied voltage. Moreover, the third peak was revealed in the case of electron injection from the wider spacer-contained contact. We notice that two first peaks

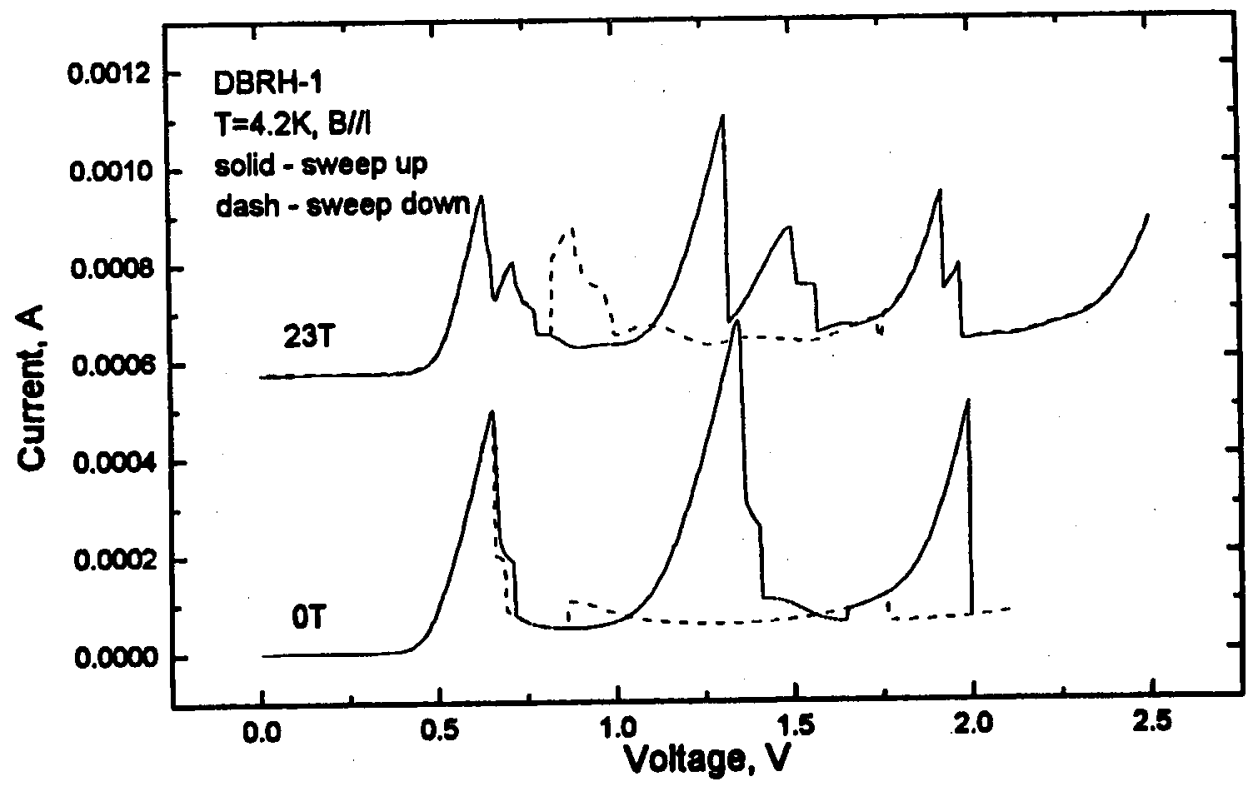

Fig. 1. Current-voltage characteristics of double-barrier heterostructure at the magnetic field parallel to the current. 


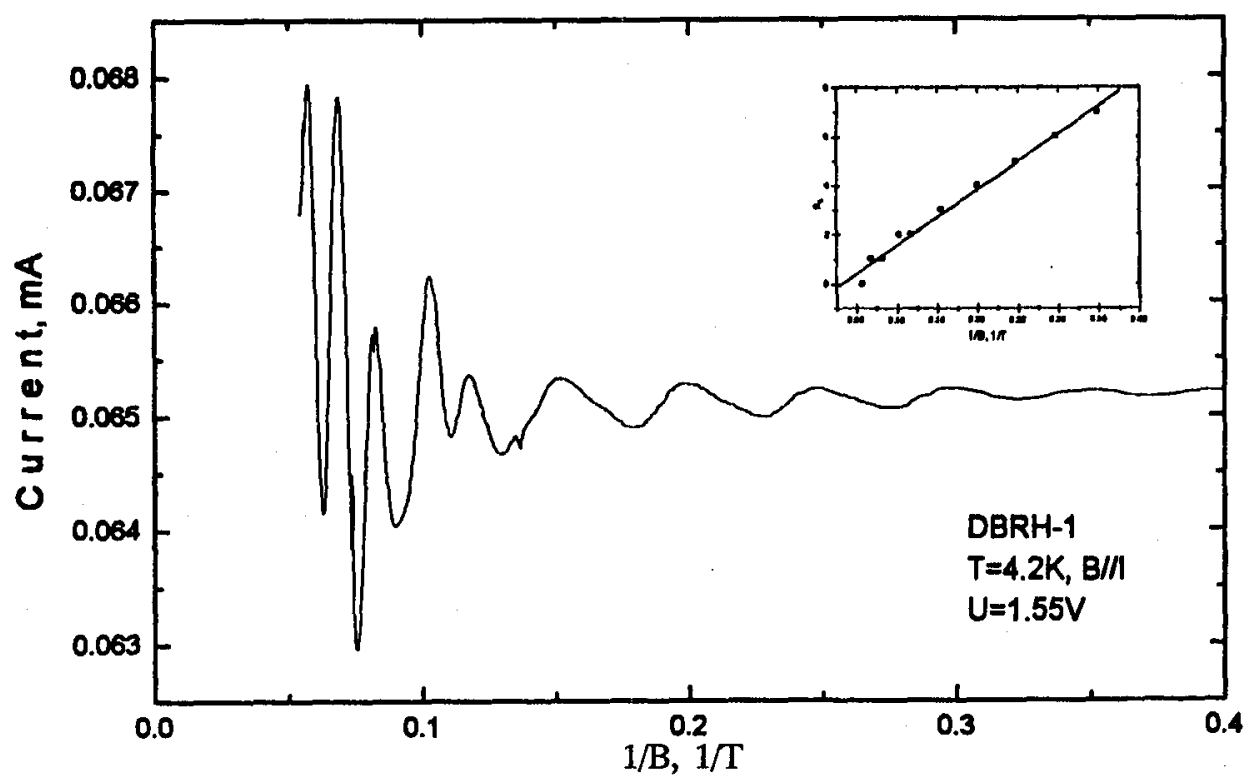

Fig. 2. Magneto-oscillations of the current measured at a fixed bias voltage. Inset dependence of Landau level's indexes on reciprocal magnetic field.

arise when the energy of ballistic electrons aligns with the states in the well, while the third peak is a result of tunneling of electrons stored in the accumulation layer. Experiments performed in a magnetic field $(B)$ confirm this assumption. Really, in the case of magnetic field applied parallel to the tunnel current the amplitude of oscillations slowly decreases with increasing $B$, but is still observable at $23 \mathrm{~T}$.

The most striking effect observed in the magnetic field parallel to the current (Fig. 1) is splitting of the resonant peaks. The effect is brightly pronounced at $B>15 \mathrm{~T}$. We have not got unambiguous explanation of this effect because different processes accompanied the resonant tunneling could be responsible for the splitting and we cannot distinguish them.

We observed an interesting effect while examining magneto-conductivity oscillations by varying the magnetic field induction at a fixed bias voltage of the device. Both the tumnel current and the differential conductance exhibit then a characteristic oscillatory structure most pronounced at high voltages (Fig. 2). This structure is periodic when displayed as a function of $1 / B$ (see the inset in Fig. 2). Moreover, we can observe a giant splitting of oscillation's maxima corresponding to Landau levels with the lowest indexes. We prescribe this effect to enhancement of $g$-factor of 2D electrons stored in the accumulation layer. Our estimates bring the value of 6.6 for the lowest Landau level that is very close to the one reported in Ref. [3].

The measurements carried out in the magnetic field applied perpendicular to the tunnel current also reveal substantial changes in both amplitude and peak position of the oscillations as a function of magnetic field. With increasing magnetic 


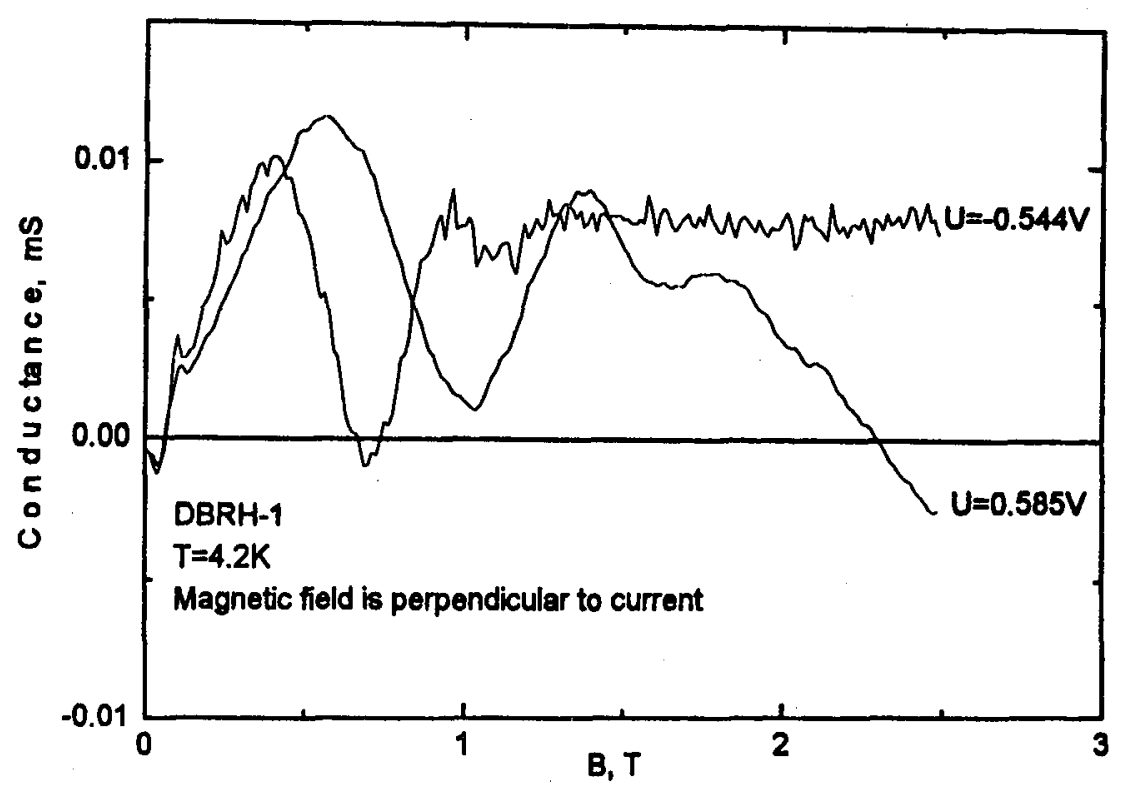

Fig. 3. Dependence of differential conductance on the magnetic field perpendicular to the current measured at a fixed bias voltage.

field the amplitude of current oscillations rapidly falls down to zero, but further rises and falls down again. This occurs due to the phase shift in the wave function of tunneling electrons [4]. It is clearly seen from Fig. 3 that the values of magnetic field $\left(B_{\mathrm{c}}\right)$, at which a shift of $2 \pi$ is reached, are quite different for the forward and reverse biases reflecting different conditions for interference of de Broglie waves. By using the values of magnetic field $B_{c}$ we could estimate the characteristic length $(L)$ corresponding to the path of ballistic motion of electrons. In the case of the forward bias we obtained the value $L$ of order of $100 \mathrm{~nm}$ for both resonant peaks, which is very close to the width of the spacer layer. The fact confirms our assumption that the interference of electron waves takes place between the interface of $n^{+}-n$ layers and emitter barrier. In the case of the reverse bias the same estimates gave us the value $L$ of $120-140 \mathrm{~nm}$, which corresponds to the width of the spacer plus depletion layer. Thus, under the reverse bias the interference of electron waves occurs in the collector region. Additional arguments, that prove our suggestion, are the calculations made in the absence of a magnetic field. Really, by using a relevant Eq. (4) of Ref. [4] we can estimate the characteristic length as a function of the bias voltage and period of oscillations. This independent analysis brings good agreement with the above-mentioned values of $L$.

\section{Conclusions}

1. Magnetotunneling experiments allow us to determine the region where de Broglie wave of electrons outgoing from the emitter or the quantum well is reflected. 
2. The spin splitting of Landau levels of 2D electron gas stored in the accumulation layer is found to be strongly enhanced by exchange interactions.

3. The characteristic lengths corresponding to path of ballistic motion of electrons were estimated.

\section{Acknowledgments}

This work was supported by the Ministry of Science and Technology of Ukraine under grant No 7.01.05/141. A.E.B. is grateful to CNRS-HMFL for the grant EEC.

\section{References}

[1] A.E. Belyaev, S.A. Vitusevich, T. Figielski, B.A. Glavin, R.V. Konakova, L.N. Kravchenko, A. Mąkosa, T. Wosiński, Surf. Sci. 361/362, 235 (1996).

[2] N. Miura, K. Yamada, N. Kamata, T. Osada, L. Eaves, Superlattices Microstruct. 9, 527 (1991).

[3] R.J. Nicholas, R.J. Hang, K. von Klitzing, G. Weimann, Phys. Rev. B 37, 1294 (1988).

[4] T. Figielski, T. Wosinski, S.A. Vitusevich, A.E. Belyaev, A. Mąkosa, W. Dobrowolski, Semicond. Sci. Technol. 12, 86 (1997). 